\title{
HARQ Implementation for Efficient MAC to MAC Video Streaming over Mobile WiMAX
}

\author{
Arathi R Shankar ${ }^{1}$, Raghavendra $\mathrm{G}^{2}$, V. Sambasiva Rao ${ }^{3}$ \\ ${ }^{1}$ Associate Professor, Department of ECE, BMSCE, Bangalore, India \\ arathi.rshekhar@gmail.com \\ ${ }^{2} \mathrm{M}$ Tech., Department of ECE, BMSCE, Bangalore, India \\ raghu.037@gmail.com \\ ${ }^{3}$ Professor, Department of ECE, PESIT Bangalore, India \\ vsrao@pes.edu
}

\begin{abstract}
IEEE $802.16 \mathrm{e}$ is the standard for Mobile WiMAX which was introduced by the IEEE committee to provide broadband access in rural and urban areas over fixed wireless channels. Mobile WiMAX is an enhanced version of IEEE 802.16 standard with mobility support. There are lots of challenges involved in the service provided by the Mobile WiMAX. Efficient data transfer between different layers of mobile WiMAX is one of the major research issues.

In this work HARQ (Hybrid Automatic Repeat Request) technique is implemented in Qualnet environment for an efficient transmission of video streaming across PHY(Physical) and MAC(Medium Access Control) layer of mobile WiMAX (Worldwide Interoperability for Microwave Access). HARQ utilizes both FEC (Forward Error Correction) and ARQ (Automatic Repeat Request) techniques to reduce the delay, jitter and to increase throughput efficiently under prescribed quality of service constraint. Different Scheduling schemes and cross layer approaches that support mobile WiMAX are analysed. mmSIR scheduler is used in this work since it allocates resources priority wise. Diffferent network quality parameters like end to end delay,average jitter,and average throughput have been analysed by using existing $A R Q$ and implemented $\mathrm{HARQ}$ techniques.Both $\mathrm{ARQ}$ and $\mathrm{HARQ}$ techniques are compared for a video application.
\end{abstract}

\section{Keywords}

HARQ,ARQ,mmSIR Scheduler,OFDM,QoS, Qualnet.

\section{Academic Discipline And Sub-Disciplines}

Engineering; Electronics \& Communication

\section{SUBJECT CLASSIFICATION}

Wireless Communication

\section{Council for Innovative Research}

Peer Review Research Publishing System

Journal: International Journal of Management \& Information Technology

Vol. 5, No. 1

editor@cirworld.com

www.cirworld.com, member.cirworld.com 


\section{APPROACH}

Following references were reviewed in order to obtain details on the general concepts of WiMAX, QoS parameters of WiMAX, HARQ, Scheduling algorithm of this work. Fundamental concepts play a major role in setting a foundation to understand the theory behind any topic. In order to get a thorough knowledge on the concepts of WiMAX the book by Dr Sunil Kumar S. Manvi,Mahabaleshwara S. Kakkasageri , "Wireless and Mobile Networks Concepts and Protocols", was referred to understand the general concepts of WiMAX, different version of WiMAX, protocol stack, properties, advantages and disadvantages.In order to get the thorough knowledge of mobile WiMAX the book by Seok Yee Tang, Peter Muller, Hamid R Sharif, "WiMAX security and quality of service", was referred to understand the Mobile WiMAX cross layer issue, its features and its Physical and MAC layer concepts.

In [11] authors have proposed a model where a cross-layer design of Adaptive Modulation and Coding (AMC) at the Physical layer with Truncated Automatic Repeat request (TARQ) and Truncated Hybrid Automatic Repeat Request (THARQ) at the Medium Access Control (MAC) layer is used, to increase the efficiency of data transmission under the prescribed quality-of-service (QoS) constraints. The authors have provided an insight in to the methods of $H A R Q$ and $A R Q$ techniques for MAC to MAC video streaming analysis, so that video streaming can be done with less packet loss and with increased efficiency of data transmission. In [5] authors have described cross-layer design and solutions for improving the performance of IEEE 802.16 WiMAX, they have defined cross layer functionalities of WiMAX and the interaction of different protocol stack. Authors have proposed a application oriented PHY-MAC cross layer model. The authors have proposed PHY-MAC Cross layer architecture for efficient data transmission, so it motivates me to work on PHY-MAC layer rather than other layers. In [13] authors have proposed maximum Signal to Interference Radio (mSIR) as scheduling algorithm and it works as follows: firstly, the BS sorts the SSs bandwidth requests in descending order according to the received SNR. Therefore, $\mathrm{mSIR}$ has the high throughput due to the best usage of slots but it does not guarantee fairness for SSs that have small SNR. In addition, authors have proposed modified maximum Signal to Interference Radio (mmSIR) which sorts SSs in descending order according to the received SNR values and if the next SS to be served has unicast opportunity in the next frame the BS does not serve this SS and serves the next SS. This gives an insight about mmSIR scheduler. In [3] authors have proposed cross layer model for efficient video transmission over mobile WiMAX by using ARQ technique. Authors have used MAC -PHY simulator for implementation. But the drawback of ARQ is fewer throughputs and more delay, this can be overcome by using HARQ technique.Literature Survey has been done on Mobile WiMAX, its advantages, its PHY layer view, MAC layer view etc. Efficient data transmission depends upon proper QoS mapping, literature has done on QoS service classes. Also survey on $H A R Q$ and $A R Q$ techniques were also done for this work.

\section{INTRODUCTION}

WiMAX stands for Worldwide Interoperability for Microwave Access, is based upon an IEEE 802.16 specifications[1]. It is a Metropolitan area network that can provide services to users within $30-50 \mathrm{~km}$ radius by using more than one station. It is a separate radio system that is designed to replace the existing broadband internet distribution systems. The main goal of WiMAX is to provide high speed internet access to home and business subscribers without wires. It provides services in two forms, one is NLOS (Non Line of Sight) service form and other one is LOS (Line of Sight) service form. In NLOS, a small antenna on the mobile unit is connected to the WiMAX tower. In NLOS the Frequency range is $2-11 \mathrm{GHz}$.

In LOS mode a fixed dish antenna points straight at the WiMAX tower from a rooftop or a pole. This connection is more reliable and stable than NLOS connection, hence it is able to send a lot of data with fewer errors. In this mode WiMAX uses a higher frequency approximately $66 \mathrm{GHz}$. It supports services such as VolP (voice over IP), TCP/IP (Transmission control/Internet protocol) applications with different quality of service (QoS) requirement. Important properties of WiMAX are,it has broad bandwidth that supports up to $134 \mathrm{Mbps}$ in $28 \mathrm{MHz}$ channel.It supports multiple services and efficiently transports IPv4,IPv6,asynchronous transfer mode (ATM), Ethernet.MAC layer offers efficient usage of spectrum.It supports adaptive modulation and space time coding.

There are different versions [1] of IEEE802.16 like 802.16a, 802.16c, 802.16d, 802.16e, and 802.16m. The physical layer of IEEE 802.16 is not suitable for lower frequency applications where non-line-of-sight (NLOS) operation is required. For this reason, the IEEE published 802.16a standard to accommodate NLOS requirement. The standard operates in licensed and unlicensed frequencies between $2 \mathrm{GHz}$ and $11 \mathrm{GHz} .802 .16 \mathrm{c}$ deals with the performance evaluation and system profiling of mandatory elements for interoperability.802.16d is an enhance version of $802.16 \mathrm{c}$ with improved QoS and less overhead. Mobility support is included in the next version $802.16 \mathrm{e}$ which supports smart antenna and MIMO (multiple input and multiple output).Next version $802.16 \mathrm{~m}$ is a future generation $4 \mathrm{G}$ standard which supports $1 \mathrm{Gbps}$ data rates and is backward compatible.

\subsection{Mobile WiMAX}

Mobile WiMAX is the mobile version of IEEE 802.16 standard, which supports mobility. The IEEE $802.16 \mathrm{e}-2005$ forms the basis for WiMAX solutions for nomadic and mobile applications and is referred to as mobile WiMAX, which works on licensed spectrum allocated in the 2.3, 2.5, 3.3, 3.5 GHz frequency bands. The IEEE $802.16 \mathrm{e}-2005$ specifications define both a physical (PHY) layer and a medium access control (MAC) layer for broadband wireless access systems. It provides high speed data connections that can be used for voice, data and video services to distances of over $30 \mathrm{~km}$.Battery life and handover are essential issues in mobile WiMAX to support mobility .In this technology two subnets in the same network and two subnets in the different network are connected to a MAN during mobility. It works in the $2.3 \mathrm{GHz}$ and 2.5 $\mathrm{GHz}$ frequency band. It adopts Orthogonal Frequency Division Multiple Access (OFDMA) for improved multipath 
performance in NLOS environments. OFDMA [2] assigns a subset of subcarriers to individual users and the transmission is simultaneous. Each OFDMA user transmits symbols using subcarriers that are orthogonal to other users. More than one subcarrier can be assigned to one user to support high data rates. This simultaneous use by multiple users results in better spectral efficiency. It uses Scalable OFDMA (SOFDMA). Mobile WiMAX systems offer scalability in both radio access technology and network architecture, thus providing a great deal of flexibility in network deployment options and service offerings. The Scalability of bandwidth is due to the fixed relationship between the occupied bandwidth and the OFDM symbol sample rate. A scalable physical layer enables standards-based solutions to deliver optimum performance in channel bandwidths ranging from $1.25 \mathrm{MHz}$ to $20 \mathrm{MHz}$ with fixed subcarrier spacing for both fixed and mobile usage models.

\subsection{Problems and Motivation}

Currently there is an explosion in the number of mobile devices capable of displaying high quality video. It is predicted that mobile traffic will increase 39 times over the five year period to 2014, which is mainly due to a significant rise in smart phone video applications. The efficiency of wireless networks needs to be optimized for the delivery of video and the convergence of voice and data. This convergence requires strict Quality of Service (QoS) for the competing user streams. Video transmission over wireless networks is challenging because of the time-varying channel quality, the high data rate and bandwidth demands of video and the latency and QoS requirements of video. The latest mobile broadband standard, IEEE 802.16, offers high user data rates and support for bandwidth hungry video applications.

Video applications demand a low Packet Error Rate (PER), and this can be achieved via the use of MAC layer Automatic Repeat Request (ARQ)[3]. However, ARQ consumes additional bandwidth and also adds to the end-to-end latency. This disadvantage of $A R Q$ is overcome by using $H A R Q$ (Hybrid ARQ). HARQ is controlled in the MAC layer by the definition of a HARQ block lifetime. Adaptive Modulation and Coding (AMC) can also be used to improve the PER by matching the QAM constellation and forward error correction coding rate to the time varying channel quality. During data exchange in wireless networks, an error in transmission can occur. Corrupted data cannot be further processed without a correction. A technique based on either Automatic Repeat Request (ARQ) or Forward Error Correction (FEC) is conventionally used to repair erroneous data in wireless networks. The ARQ is backward mechanism that uses a feedback channel for the confirmation of error-free data delivery or to request a retransmission of corrupted data. This method can increase a network throughput if radio channel conditions are getting worse. On the other hand, the ARQ method increases the delay of packets due to the retransmission of former unsuccessfully received packets. The FEC can increase user's data throughput over the channel with poor quality despite the fact that additional redundant bits are coded together with users' data at the transmitter side. The method combining both above mentioned methods is called Hybrid ARQ (HARQ), which is implemented in this work in the MAC layer for efficient video transmission.

\section{PROPOSED BLOCK DIAGRAM}

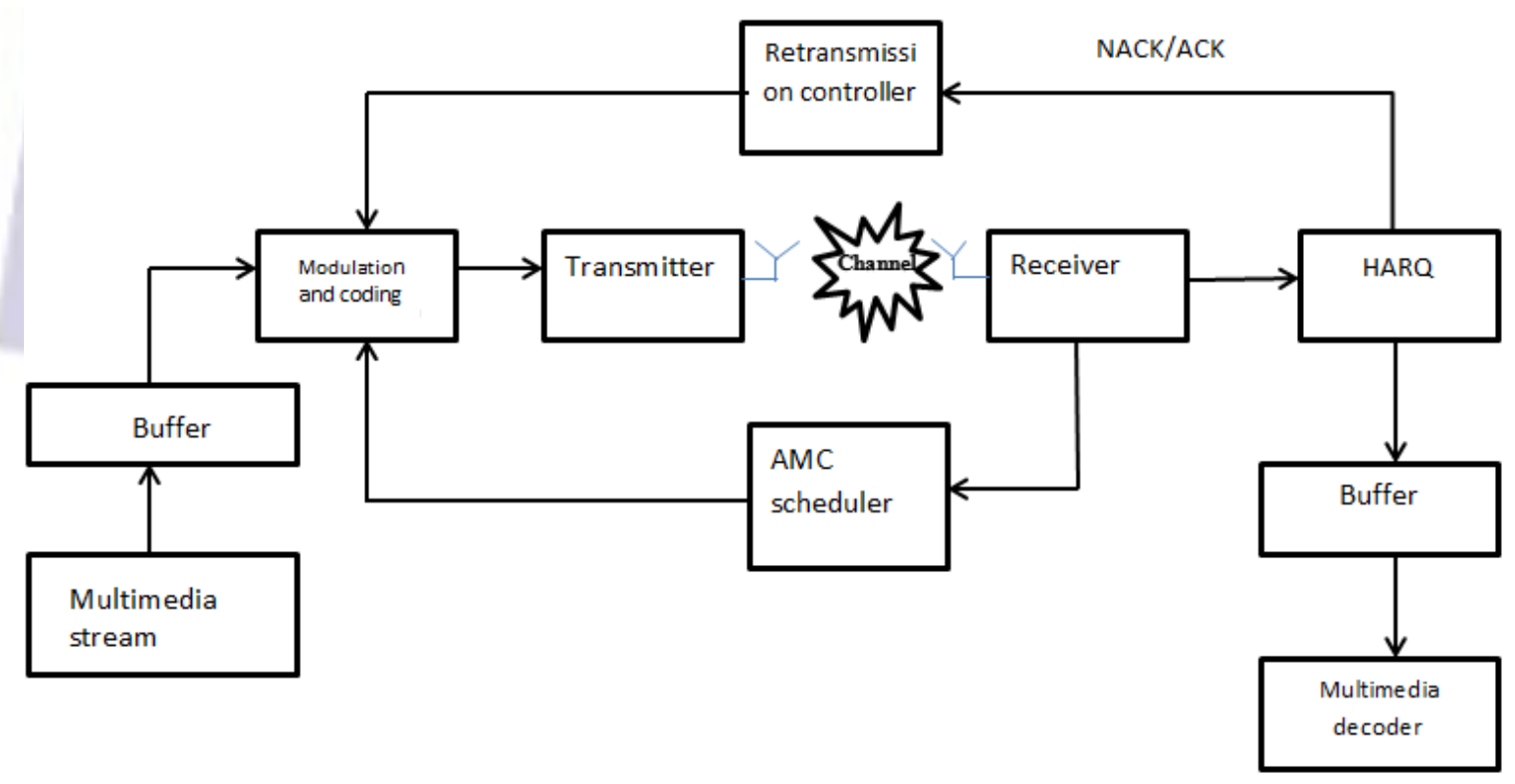

Figure 1: Proposed Block Diagram

The proposed block diagram model is shown in the above Figure1. Video application is used here. The network has been simulated using Qualnet simulator version 6.0. It doesn't support real time video application, so we have taken only video and this can be analysed as the number of frames transmitted, number of frames received, number of data transmitted, number of data received etc. First stream of video data is buffered using a finite-length queue (buffer), which is implemented at the transmitter and operates in a first- in-first-out mode [10]. The queue feeds data in to Modulation and 
coding unit which is controlled by the AMC controller/scheduler at the transmitter. Channel is considered as AWGN. HARQ performs error correction and detection mechanism. Regenerative controller block has controlling circuit which enable to retransmit the lost data depending upon the reception of NACK/ACK signal from the receiver .At the wireless link (PHY), multiple transmission modes are available, with each mode representing a pair of a specific modulation format, and a Forward Error Correcting (FEC) code. The AMC design considered assigns the exact mode based on channel estimation at the receiver. The AMC scheduler determines the modulation-coding pair (mode), which is sent back to the transmitter through a feedback channel, to update the transmission mode. There are different schedulers available for the WiMAX, some of them are known one and some are designed by the user according to the proposed model. In this work scheduler will be used which allocate the resource according to priority wise. Highest priority is given to the one which has highest Signals to Interference Ratio (SIR). Some multimedia decoders are used at the receiver to decode the bits. The decoded bit streams are mapped to packets, which are pushed upward to the data link layer.

\section{$2.1 \mathrm{mmSIR}$ Scheduler}

Scheduler used in this work is mmSIR (maximum modified Signal to Noise Ratio) scheduler[14]. In this scheduler BS allocates symbols for the UGS, then rtPS and finally BE connections. For the allocation of symbols for rtPS connection, the BS allocates periodical unicast request opportunities and then, according to these requests, the symbols needed for the rtPS connections. If the BS allocates unicast request opportunities and resource grants for rtPS connections in the same frame, the BS cannot immediately take into account the new length of the uplink data connection of the subscriber. The reason is that the BS allocates symbols for rtPS connections before receiving the latest unicast bandwidth request.Moreover, the mSIR scheduler serves those subscribers having the highest SIR at each frame. So, subscribers having a slightly smaller SIR may be not served and then the mean delay to deliver data increases. We need to modify the SIR scheduler in order to decrease the delay. In our proposed model the BS only serves the subscribers that do not have unicast request opportunities in the same frame. The resulting scheduler is called the modified maximum Signal-toInterference Ratio (mmSIR) scheduler. The main steps of this scheduler are shown in figure 2.

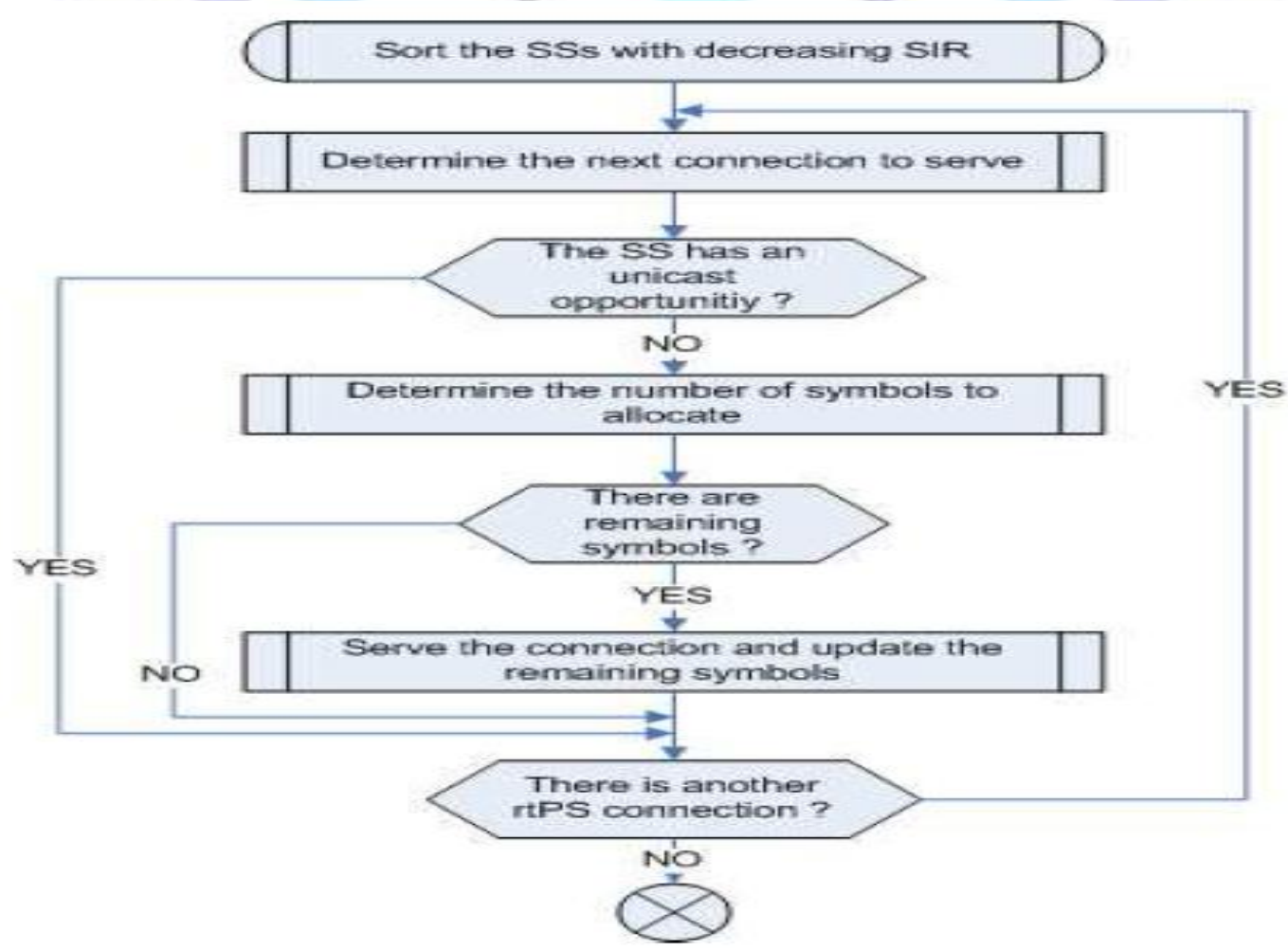

Figure 2: $\mathrm{mmSIR}$ Scheduler

To improve the video quality efficiency the authors have proposed MAC/PHY cross layer issue(as shown in fig 1) that enhances the received video quality over mobile WiMAX while maintaining required QoS constraint. The authors have estimated QoS parameter like throughput, end to end delay and average jitter using ARQ techniques .On doing this there is a decrease in the packet loss rate and increase in the video quality with increase in delay and jitter,this can be overcome by using $H A R Q$ technique. We the authors have implemented $H A R Q$ techniques instead of $A R Q$ in the $\mathrm{MAC} / \mathrm{PHY}$ cross layer an increase in the throughput is obtained and hence efficiency in the video quality (in terms of number of frames ) received with decrease in the delay and jitter.

There are different cross layer approaches used for the cross layer implementation of WiMAX ,but in this work an integrated approach has been implemented. This approach takes an optimal decision based on all information, parameters, and requirements, which are received from the different layers. Despite other approaches, all layers can send their required quality, and there is not only a unique layer to dictate the quality to the other layers [14]. Therefore, this 
approach should provide different requirements for different layers; hence this approach is best suitable method for the current application. However, this approach needs a real-time optimization method to provide the optimal layer values. We authors used the network simulator Qualnet 6.1 to simulate transmission of video traffic across PHY/MAC layer over WiMAX for two different scenarios, one by disabling $A R Q$ and other one by enabling $A R Q$. In the case where $A R Q$ is incorporated, more delay and jitter exist in the received data but decrease in the packet loss rate and thereby increase in the throughput. Since $H A R Q$ uses both $A R Q$ and FEC, we obtain further reduction in the packet loss rate and increase the video quality.

\section{SIMULATION}

Simulation was carried out using Qualnet Simulator. Mobile WiMAX scenario was created in Qualnet environment using nodes(radio) and subnet(cloud)as shown in figure 3.

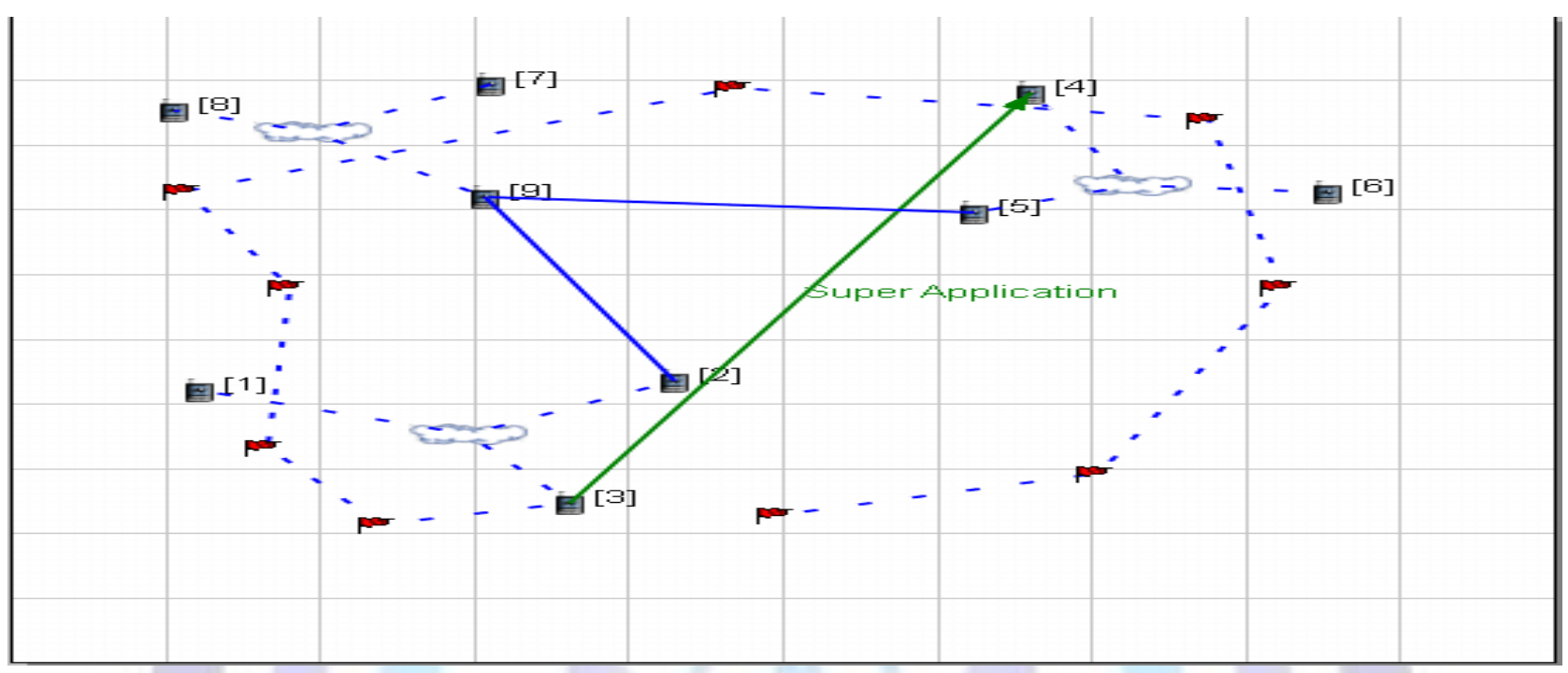

Figure 3: Mobile WiMAX Scenario created for HARQ implementation

Simulation setup is shown in figure 3 .Three homogeneous networks are considered. Each network is connected with 3 nodes. Node 2, 9, and 5 are base stations connected by wired line (It can also be connected with wireless link). Super application is connected between node 3 and node 4. Video Traffic is present in the Super application as shown in fig.4.Different encoding scheme are given in the Super application, we have taken H.263 encoding scheme. Since we are sending data from source node 3 to destination node 4 , no need to specify source and destination port. It will automatically assume source port as 3 and destination port as 4 .Uniform time distribution is considered because packets reception will uniformly vary with respect to time. We can also use exponential time distribution, but the efficiency will be very less as compared to uniform distribution .Enable fragmentation could be used only in the case of audio application, since Qualnet support default fragmentation for video application.

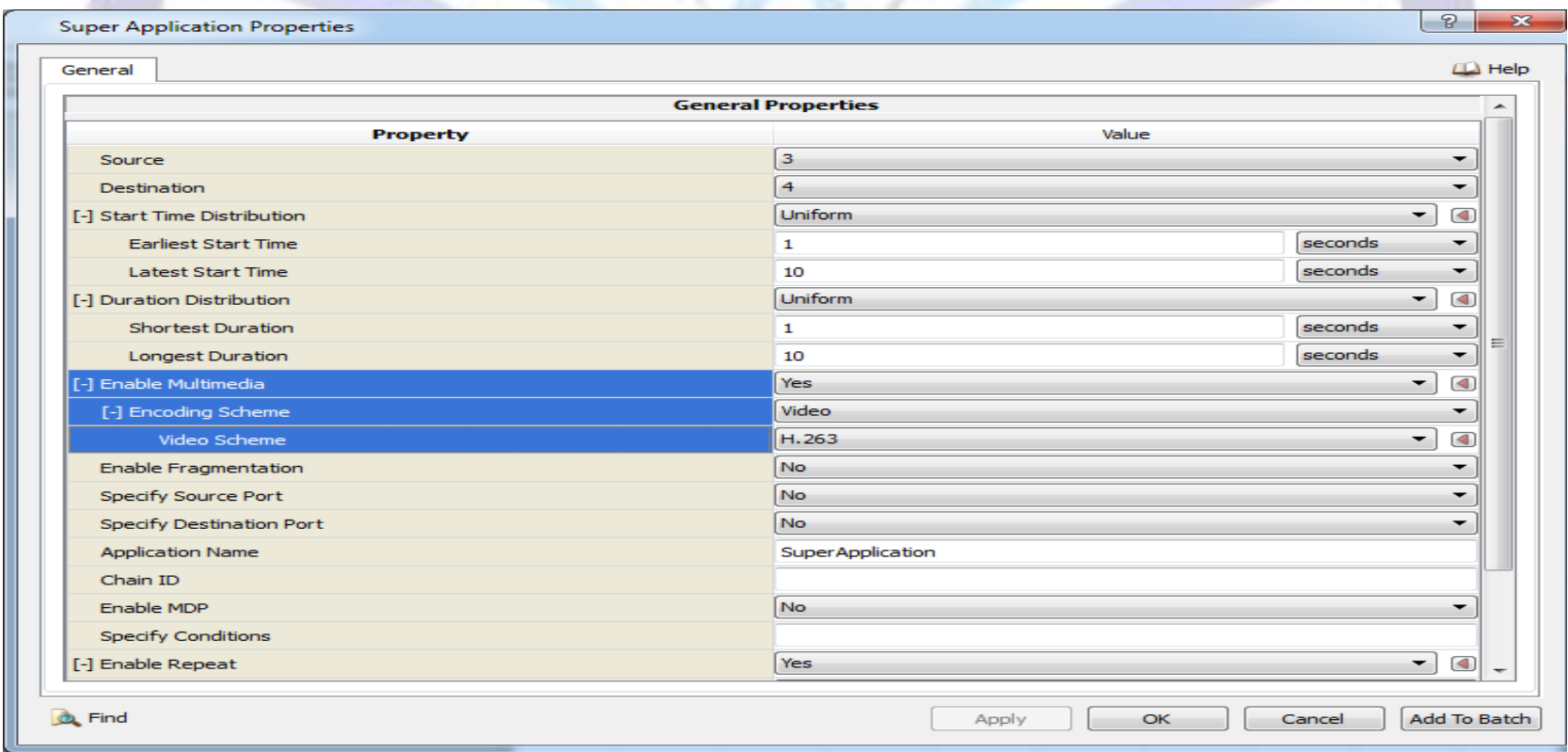

Figure 4:Super Application properties in Qualnet Simulator 
HARQ techniques is implemented in the MAC layer along with its associated parameter like HARQ window size, HARQ retry timeout, HARQ retry count, $H A R Q$ syn loss interval, $H A R Q$ block size etc. as shown in the figure 5 . HARQ window size indicates maximum bytes of data that can transfer in one packet. HARQ retry timeout is the retransmission time of the lost or error packet. If the receiver doesn't receive the lost or error packet within this time from the source, that error packet will be discarded. $\mathrm{HARQ}$ retry count is used to indicate the maximum number of error packet take can be retransmitted.MAC propagation delay is the time taken to transmit a packet of data from source to destination, which is taken as 1 millisecond in this simulation environment.

MAC layer subnet properties of Qualnet simulator do mention about ARQ but not HARQ . Novelty of the work is to implement HARQ in Qualnet.

\begin{tabular}{|c|c|c|c|c|c|c|c|c|c|}
\hline \multicolumn{8}{|c|}{ Wireless Subnet Properties (Wireless Subnet 190.0.30) } & \multicolumn{2}{|c|}{\begin{tabular}{|l|l|} 
Q & $x$ \\
\end{tabular}} \\
\hline General & Physical Layer & MAC Layer & Network Layer & Routing Protocol & Router Properties & File Statistics & & 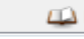 & Help \\
\hline \multicolumn{4}{|c|}{ Wait UCD Timeout Interval } & 25 & & & seconds & - & \multirow[t]{5}{*}{-} \\
\hline \multicolumn{4}{|c|}{ Scrvec Flow Timcout Interval } & 15 & & & seconds & - & \\
\hline \multicolumn{4}{|c|}{ Enable Packing } & No & & & & - & \\
\hline \multicolumn{4}{|c|}{ Ranging Type } & Normal & & & & - & \\
\hline \multicolumn{4}{|c|}{ Contention-based Bandwidth Request Type } & Normal & & & & - & \\
\hline \multicolumn{4}{|c|}{ [-] Enable Mobility Mode $(802.16 e)$} & Yes & & & & $\rightarrow \square$ & \\
\hline \multicolumn{4}{|c|}{ Neighbor BS Scanning RSS Trigger (dBm) } & -76.0 & & & & & \\
\hline \multicolumn{4}{|c|}{ Handover RSS Trigger (dBm) } & -78.0 & & & & & \\
\hline \multicolumn{4}{|c|}{ Handover RSS Margin (dB) } & 1.0 & & & & & \\
\hline \multicolumn{4}{|c|}{ Enable Sleep Mode } & No & & & & - & \\
\hline \multicolumn{4}{|c|}{ Enable Idle Mude } & No & & & & - & \\
\hline \multicolumn{4}{|c|}{ [-] Enatle HARQ } & Yes & & & & (4) & ( \\
\hline \multicolumn{4}{|c|}{ HARQ Window Size } & 1024 & & & & (4) & $\equiv$ \\
\hline \multicolumn{4}{|c|}{ HARQ Retry Timeout (frames) } & 4 & & & & & \\
\hline \multicolumn{4}{|c|}{ HARQ Retry Count } & 2 & & & & & \\
\hline \multicolumn{4}{|c|}{ HARQ Sync Loss Interval (frames) } & 32 & & & & & \\
\hline \multicolumn{4}{|c|}{ HARQ Receiving Purge Timeout Interval (frames) } & 28 & & & seconds & $\rightarrow \square$ & ( \\
\hline \multicolumn{4}{|c|}{ HARQ Block Size (bytes) } & 64 & & & & & \\
\hline \multicolumn{4}{|c|}{ MAC Propagation Delay } & 1 & & & milli-seconds & $-\square$ & ( \\
\hline \multicolumn{4}{|c|}{ tnable Promiscuous Mode } & No & & & & - & ] \\
\hline \multicolumn{4}{|c|}{ Enable LLC } & No & & & & - & - \\
\hline Q. Find & & & & & Apply & OK & Cancel & Add To $E$ & Batch \\
\hline
\end{tabular}

Figure 5: Wireless Subnet Properties where HARQ technique is implemented

Scheduler plays a crucial role in the WiMAX network to increase the efficiency of the data transmission.mmSIR Scheduler is used in this work. This Scheduler is implemented in the Network layer under the subnet properties 'IP output queue scheduler', number of IP output queue is taken as 3 as shown in figure 6. .. There are different schedulers which are already present in the Qualnet environment. But among all those schedulers, mmSIR Scheduler is the one which gives more efficiency in terms of average throughput. In the QoS configuration we can configure required QoS strategies.Video application require rtPS(Real Time Polling Service) service class and this can be chosen in the QoS configuration property. In Qualnet priority is given to different QoS service class. 1 is given to UGS service class,2 is given to rtPS service class, 3 is given to ertPS service class, 4 is given to nrtPS, and 5 is given to Best Effort service class.

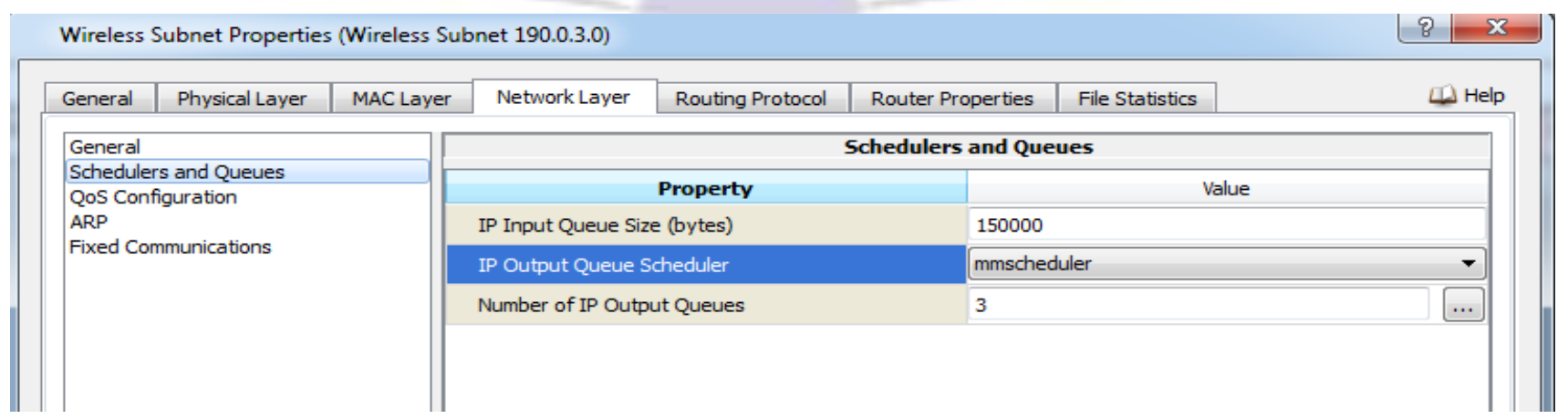

Figure 6: Wireless Subnet properties where mmSIR Scheduler is implemented 
Table 1. Simulation parameter

\begin{tabular}{|c|c|}
\hline Parameter & Value \\
\hline Carrier Frequency & $2.4 \mathrm{GHz}$ \\
\hline Channel Bandwidth & $20 \mathrm{MHz}$ \\
\hline FFT size & 2048 \\
\hline Cyclic prefix factor & $20 \mathrm{dBm}$ \\
\hline Transmission Power & $1024 \mathrm{bytes}$ \\
\hline HARQ window size & 64 bytes \\
\hline HARQ Block size & 4 frames \\
\hline HARQ retry timeout & 2 \\
\hline HARQ retry count & 32 \\
\hline HARQ sync loss interval & \\
\hline
\end{tabular}

Visual C++ programming language is used for coding. All codes are dumped in the Microsoft Visual Studio 2008 software combined with the existing code of WiMAX. The implemented HARQ techniques with associated parameters have appeared in the Qualnet environment.

\section{RESULT AND DISCUSSION}

Mobile WiMAX scenario (shown in figure 3) is simulated first by enabling ARQ techniques, results obtained from the simulation were noted down and the graphs were plotted. After this HARQ technique is implemented in the Qualnet. The same mobile WiMAX scenario (as shown in figure 3) is used to simulate the implemented HARQ technique. The results obtained from this are again noted down and the graphs are plotted. From the comparison of both the results we come to the conclusion that HARQ technique is more efficient than $A R Q$ technique.

\subsection{Implementation of $A R Q$ technique}

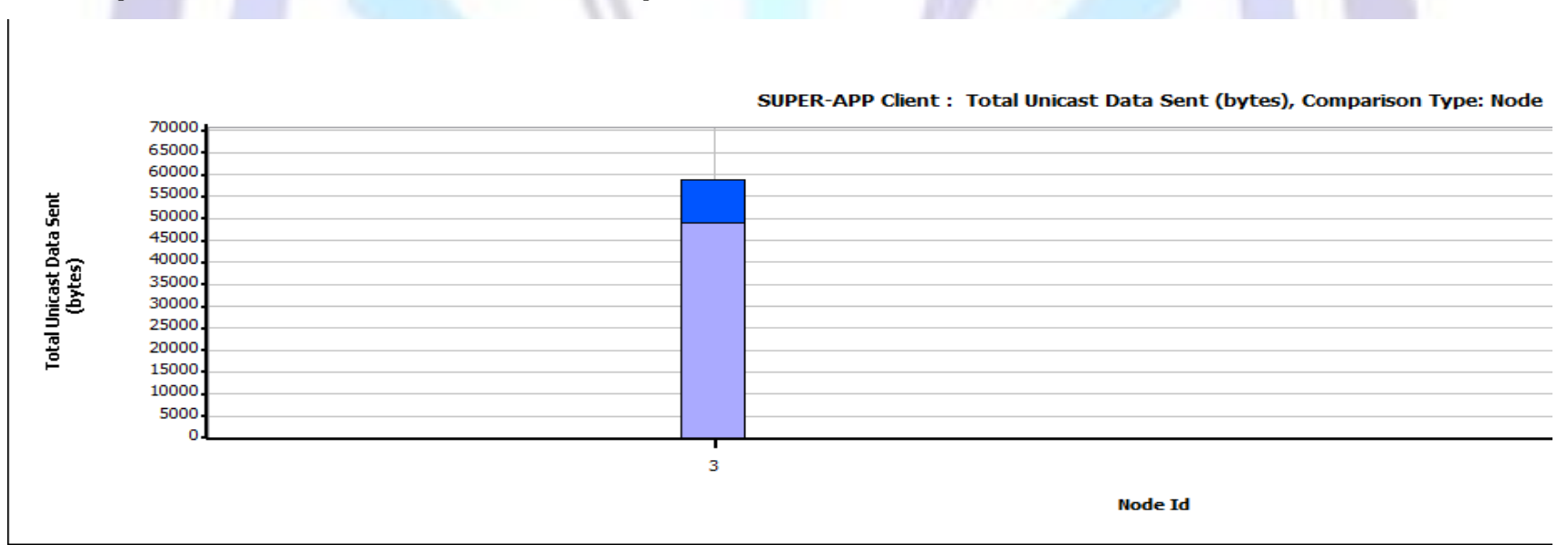

Figure 7: Total Unicast Data sent using ARQ (59200 bytes)

Figure 7 shows total data sent from the node 3 of one network to node 4 of another network using unicast data transmission. 
SUPER-APP Server : Total Unicast Data Received (bytes), Comparison Type: Node

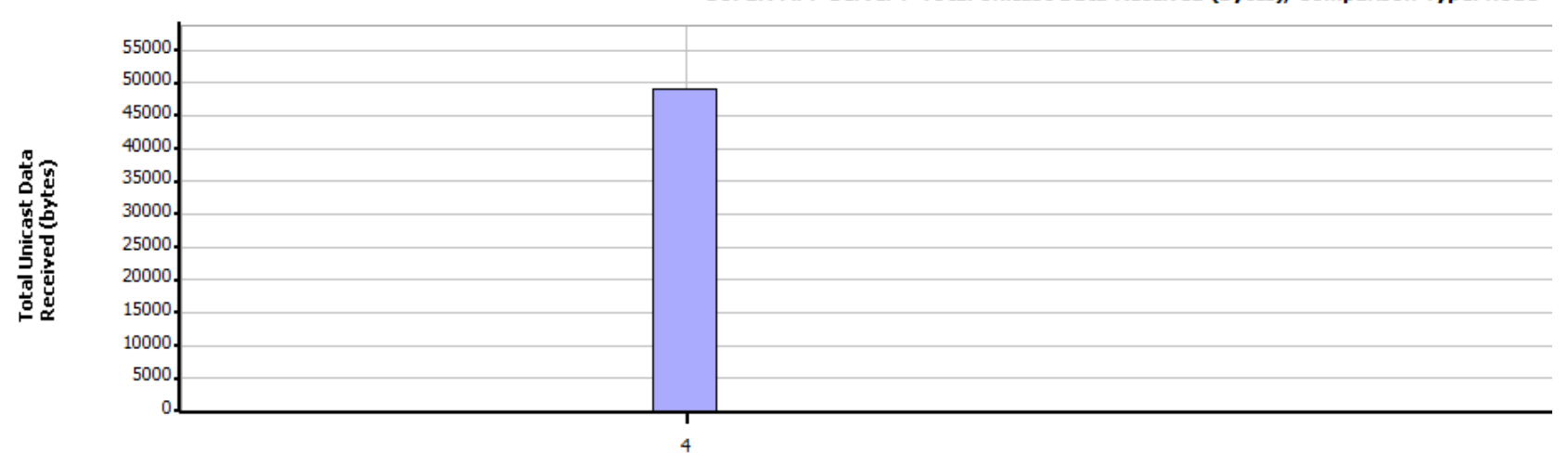

Node Id

Figure 8: Total Unicast Data received using ARQ (49120 bytes)

Figure 8 depicts total data received at node 4 .If we calculate the ratio of number of data received to the number of data transmitted, the ratio will be $82.97 \%$.

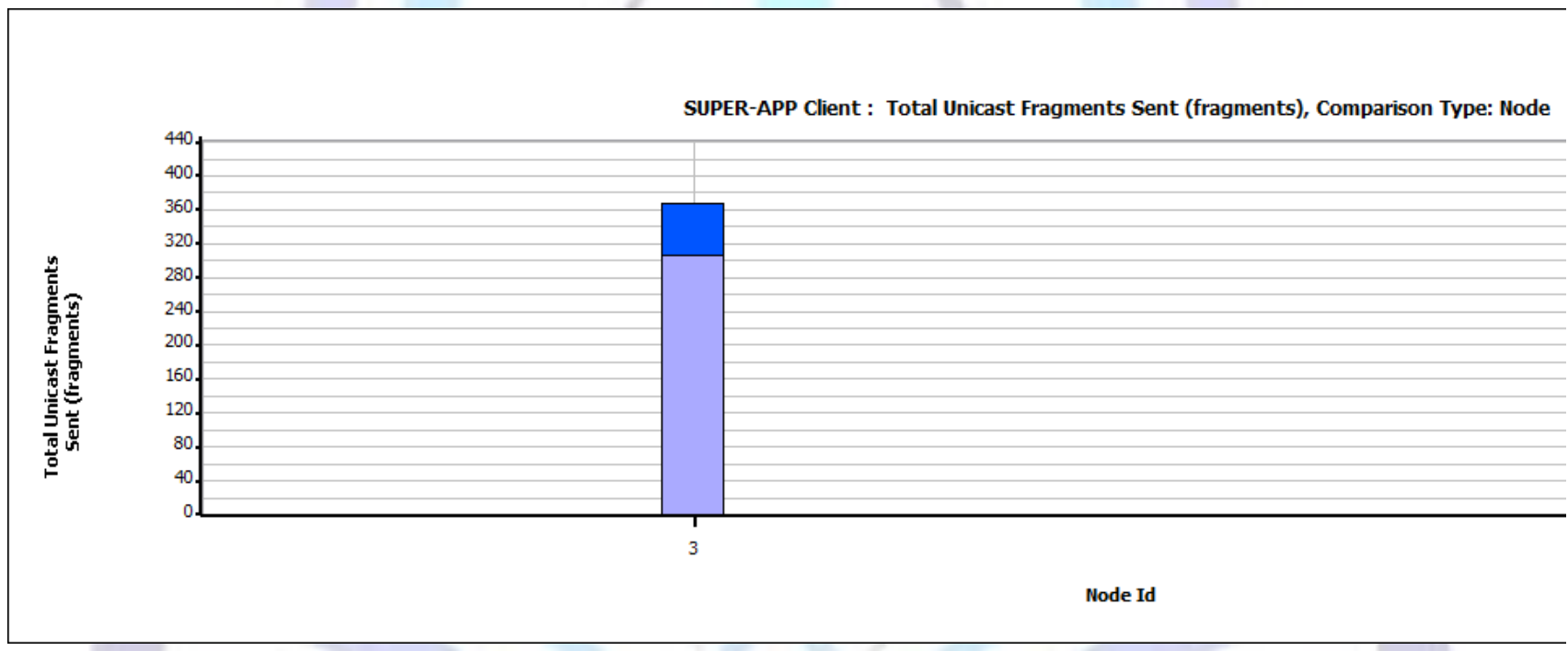

Figure 9: Total Unicast Fragments sent using ARQ (370 frames)

Total number of Fragments or frames sent from node 3 to node 4 is shown in figure 9. The two colour marks in the above figure represent two different ports through which frames are transmitted. Dark blue colour represent port 1024, whereas light bluish represent port 1025.

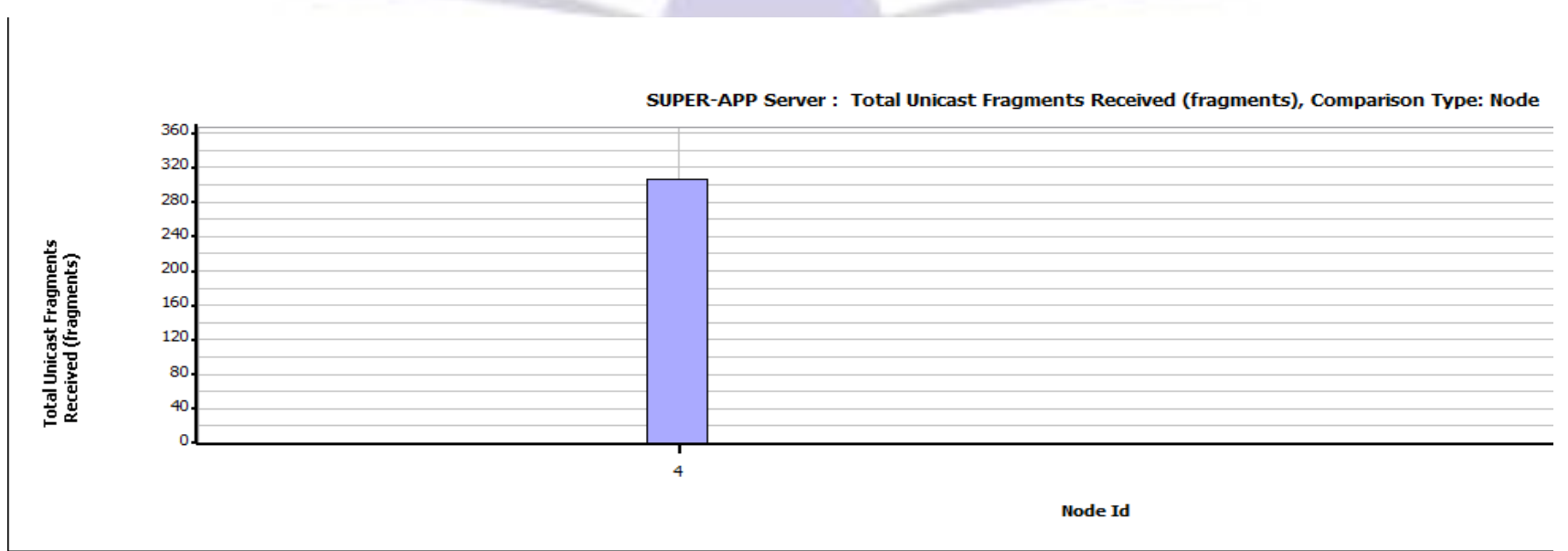

Figure 10: Total Unicast Fragments received using ARQ (307 frames) 
Figure 10 depicts total fragments or frames received at node 4. Ratio of total frames received to the total frames sent is $82.97 \%$.

Figure 7 - Figure 10 indicates the ration of data sent to data received as well as ratio of frames sent to frames received by using $A R Q$ technique, which is obtained as $82.97 \%$, this can be increased further by using $H A R Q$ technique.

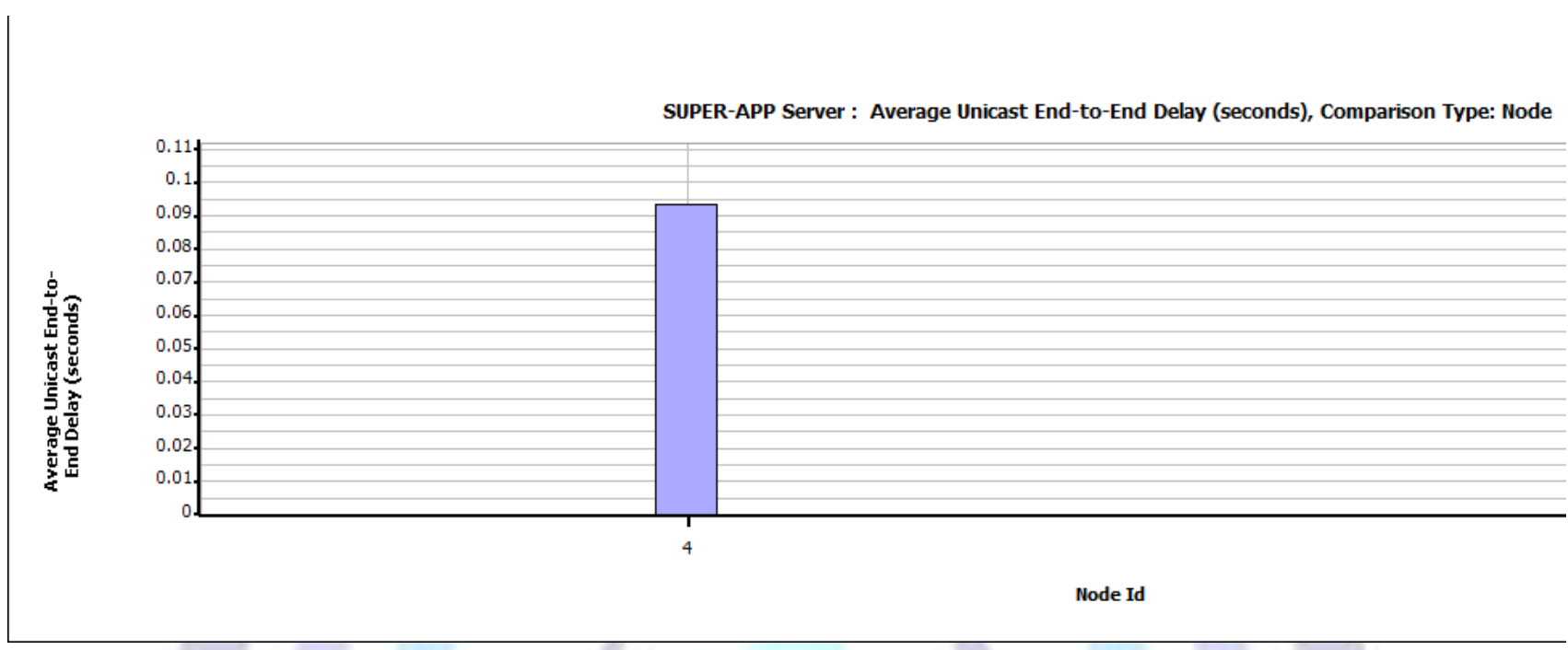

Figure 11: Average End to End Delay using ARQ (0.0935 sec)

End to End delay achieved by using $A R Q$ technique to transmit video data over mobile WiMAX network is shown in figure 11.It take $0.0935 \mathrm{sec}$ to transmit one packet of data from source to destination.

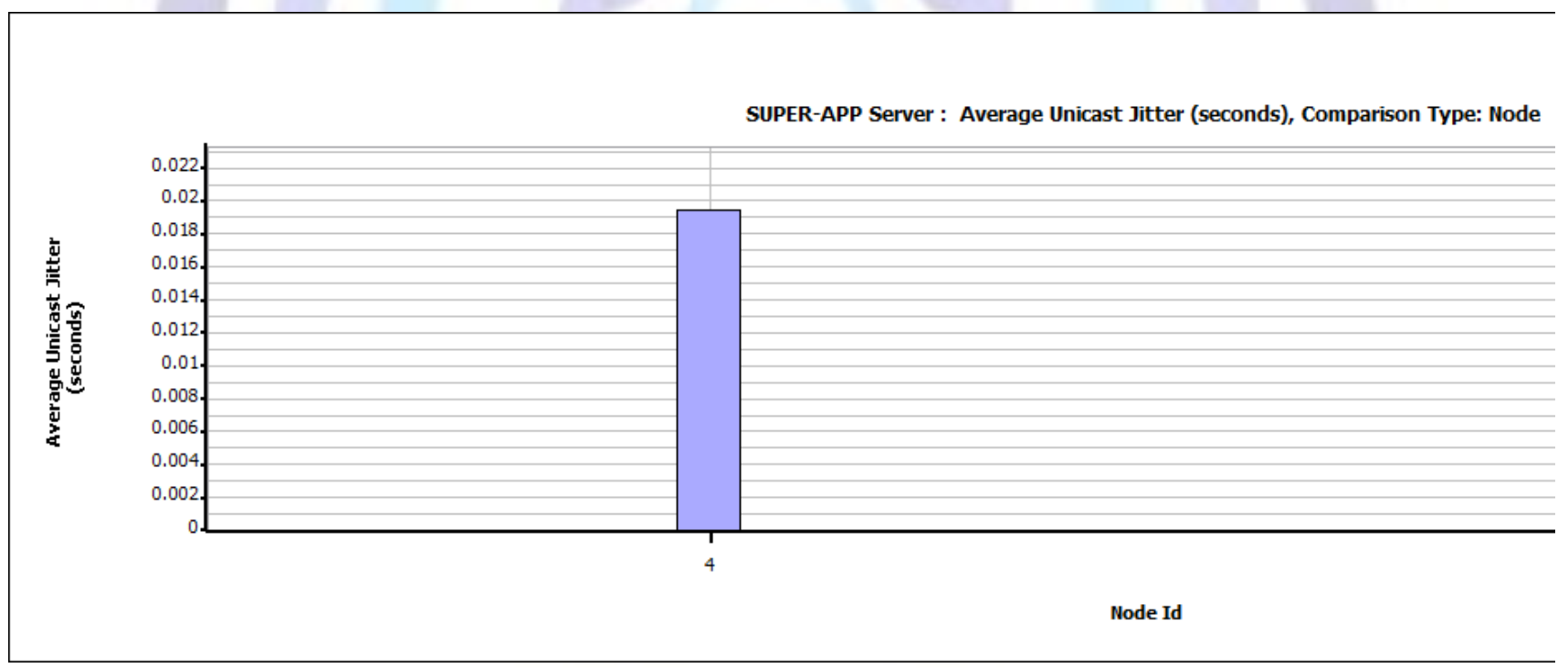

Figure 12: Average Jitter using ARQ (0.01947 sec)

Figure 12 shows average jitter obtained after simulation of WiMAX network. The variation in packet delay is called as jitter. We have got $0.01947 \mathrm{sec}$ delay variation by using $A R Q$ technique. 


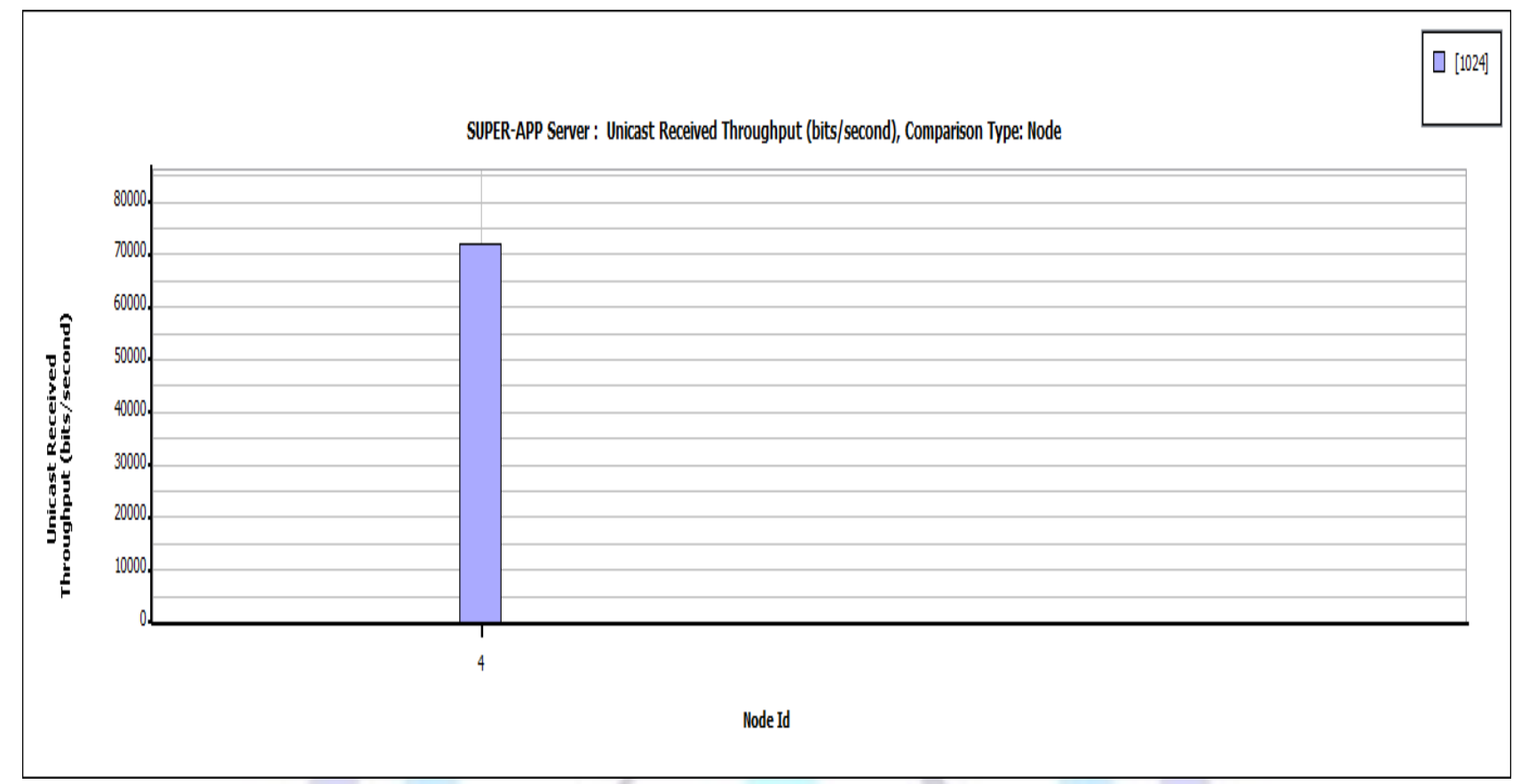

Figure 13: Throughput using ARQ (72112.7 bits/sec)

Average throughput is the average rate of successful data packet delivery over a communication channel. This data may be delivered over a physical or logical link, or pass through a certain network node. The throughput is usually measured in bits per second (bit/s or bps), and sometimes in data packets per second or data packets per slot. Here we have obtained a throughput of 72112.7 bits/sec by using $A R Q$ technique.

\subsection{Implementation of HARQ Technique}

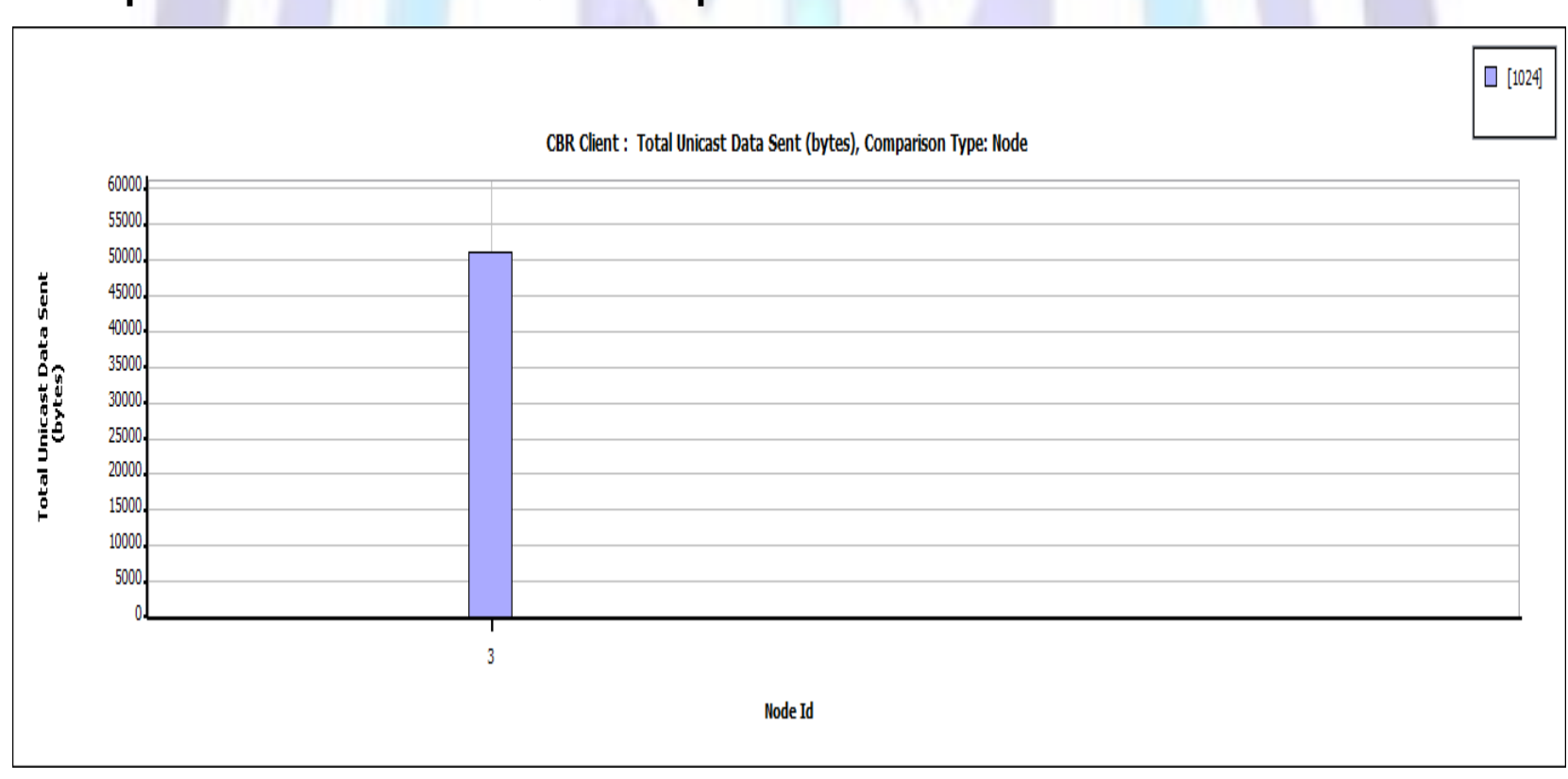

Figure 14: Total Unicast data sent using HARQ (51200 bytes)

Figure 14 shows total data sent from the node 3 of one network to node 4 of another network using $H A R Q$ technique. Totally 51200 bytes of data is transmitted over WiMAX network. 


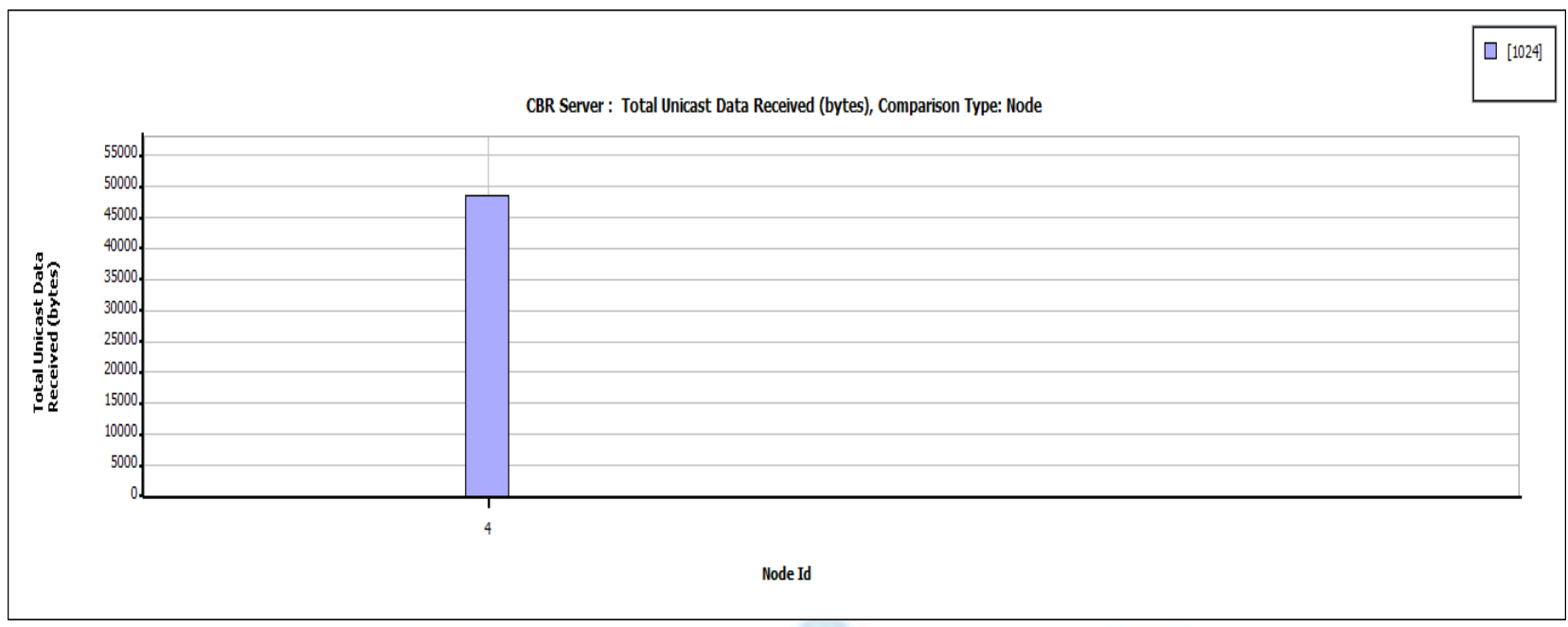

Figure 15: Total unicast data received using HARQ (48640 bytes)

Figure 15 depicts total data received at node 4. The ratio of number of data received to the number of data transmitted is $95 \%$.

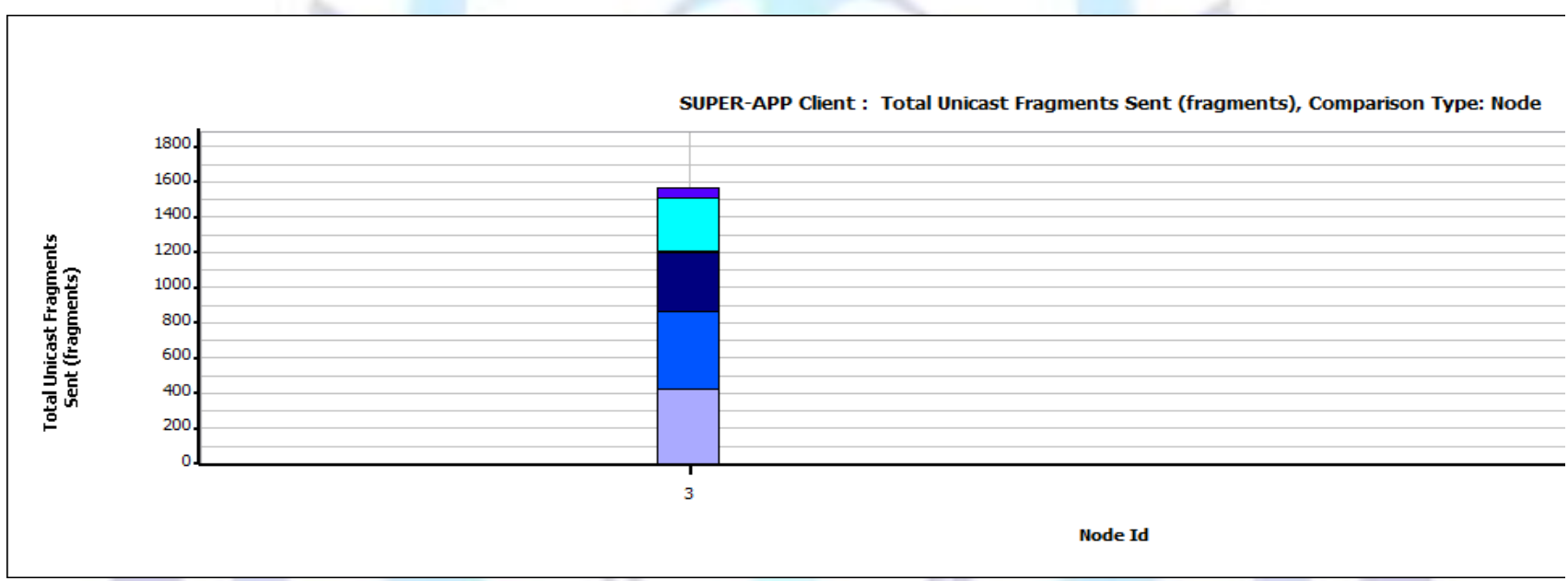

Figure 16:Total Unicast Fragments sent using HARQ (1580 frames)

Figure 16. depicts total frames transmitted from source to destination. Totally 1580 frames of video application are transmitted over WiMAX network.

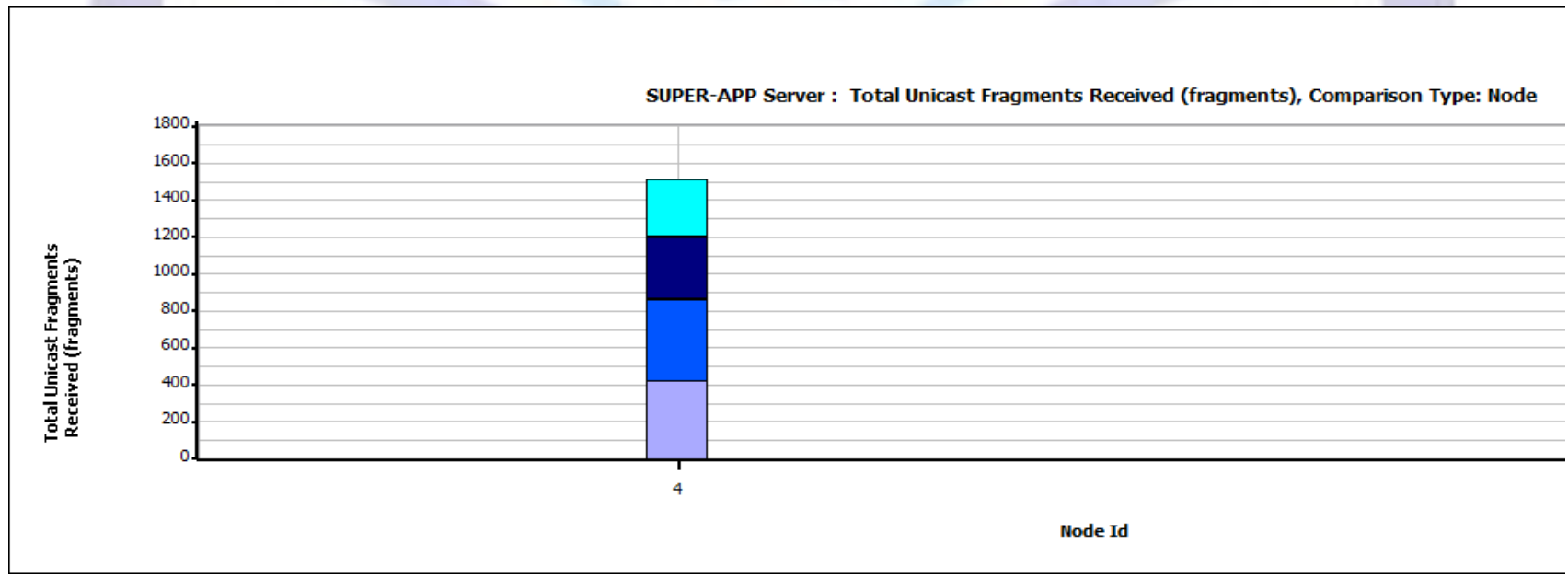

Figure 17:Total Unicast Fragments received using HARQ (1510 frames)

Figure 17 depicts total fragments or frames received at node 4.Ratio of total frames received to the total frames sent is $95.56 \%$, which is approximated to $95 \%$.By comparing HARQ with the ARQ in terms of number of data packet received and 
the number of frame received, $\mathrm{HARQ}$ is more efficient than $\mathrm{ARQ}$ for video application .Because in $\mathrm{HARQ} 95 \%$ of the data transmitted will be received at the receiver, whereas in case of ARQ only $82.97 \%$ of transmitted data is received.

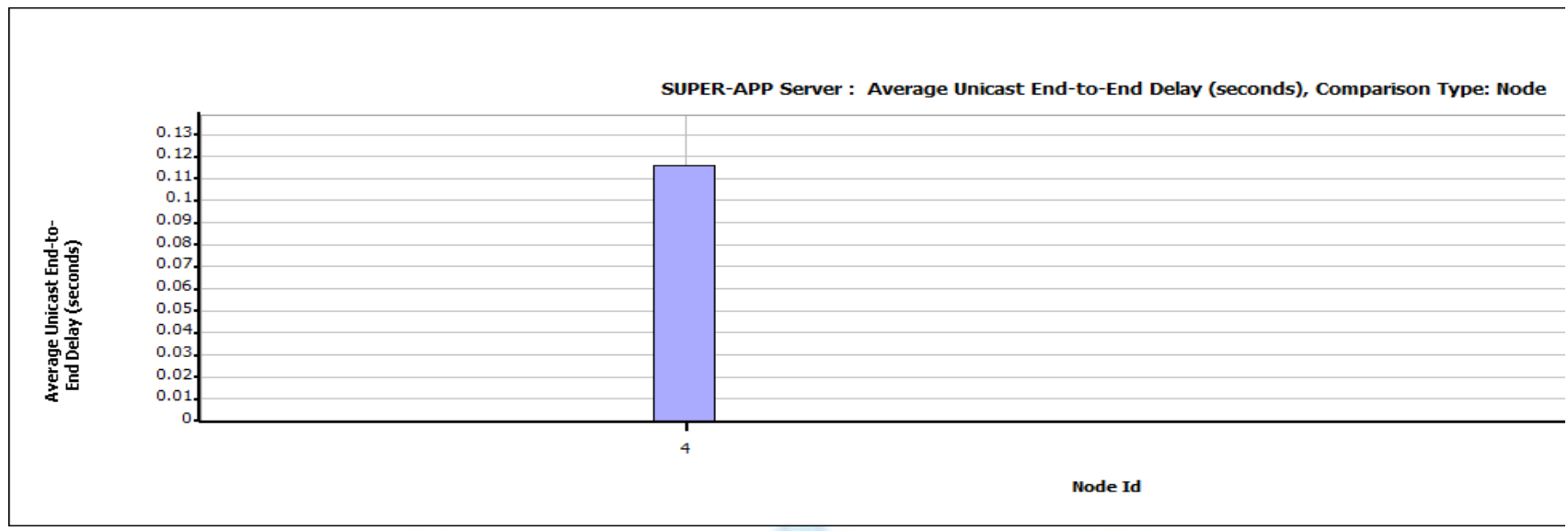

Figure 18: Average End to End delay using HARQ (0.1816 sec)

End to End delay achieved by using HARQ technique to transmit video data over mobile WiMAX network is shown in figure 18.It take $0.1816 \mathrm{sec}$ to transmit one packet of data from source to destination.

By comparing figure 18 with figure 11 , end to end delay will be more in $H A R Q$ compare to $A R Q$, this is due to the time taken for the retransmission of lost or error packet. HARQ will take more time to receive correct packet, hence it will give good throughput efficiency.

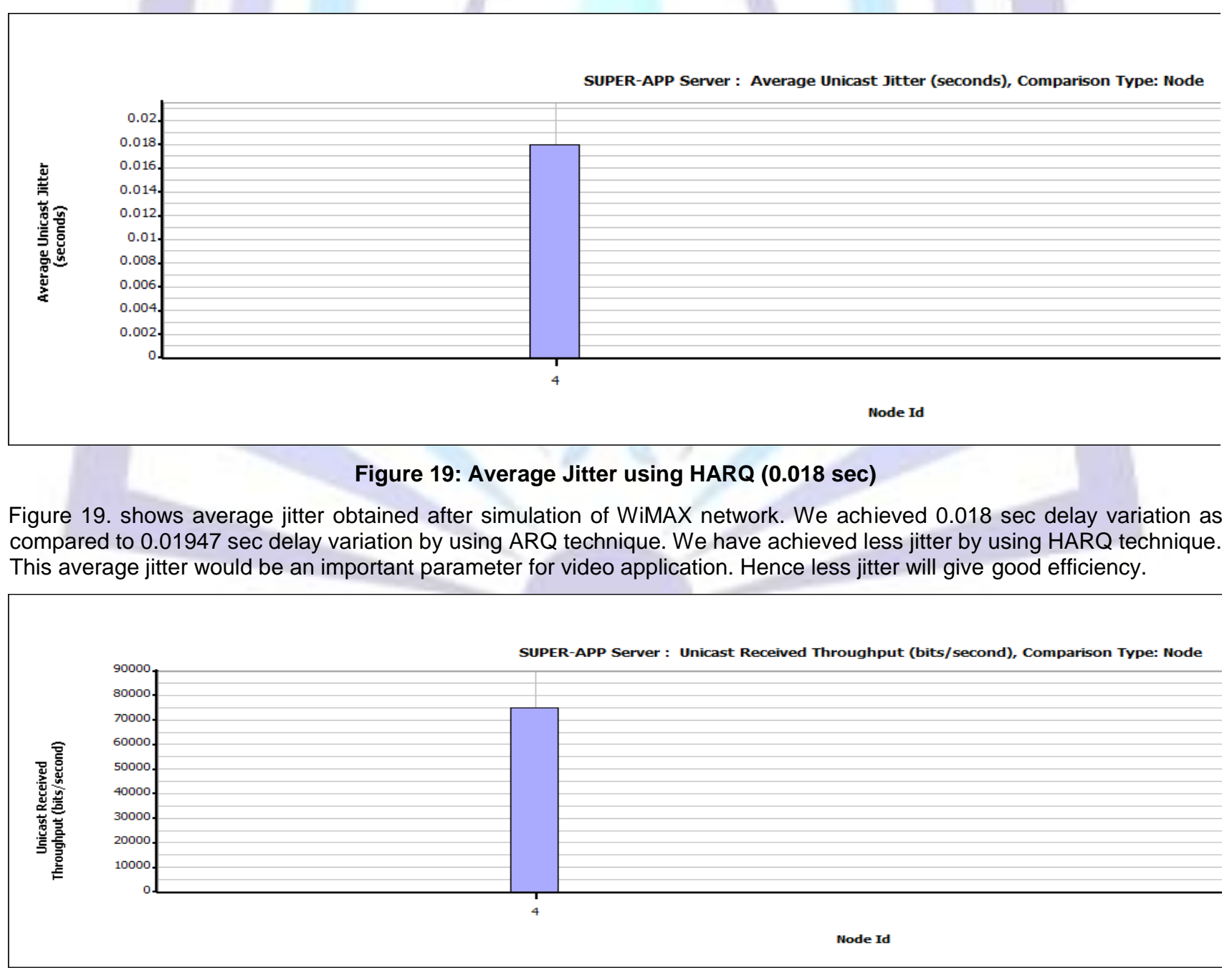

Figure 20: Throughput using HARQ (75230 bits/sec)

Figure 20 depicts the average throughput obtained in bits/sec after simulation using HARQ technique. 
We compared figure 20 with the figure 13 , by using HARQ we achieved $75230 \mathrm{bits} / \mathrm{sec}$ throughput, whereas by using ARQ we achieved only 72112.7 bits/sec throughput. So the throughput will be more in HARQ than ARQ.

Table 2. Comparison of $H A R Q$ and $A R Q$

\begin{tabular}{|c|l|c|c|}
\hline SI no. & \multicolumn{1}{|c|}{ Parameters } & ARQ & HARQ \\
\hline 1 & Total Unicast Data sent & 59200 & 51200 \\
\hline 2 & Total Unicast Data received & 49120 & 48640 \\
\hline 3 & Total Fragments Sent & 370 & 1580 \\
\hline 4 & Total Fragments received & 307 & 0.1816 \\
\hline 5 & End to End delay (in sec) & 0.0935 & 0.018 \\
\hline 6 & Average Jitter (in sec) & 0.01947 & 75230 \\
\hline 7 & Throughput (in bits/sec) & 72112.7 & 1510 \\
\hline
\end{tabular}

Table 2.shows the comparison result of $H A R Q$ with $A R Q$, this comparison indicates that HARQ can be efficiently used for MAC to MAC video transmission over mobile WiMAX both for real time and distributed time video application.

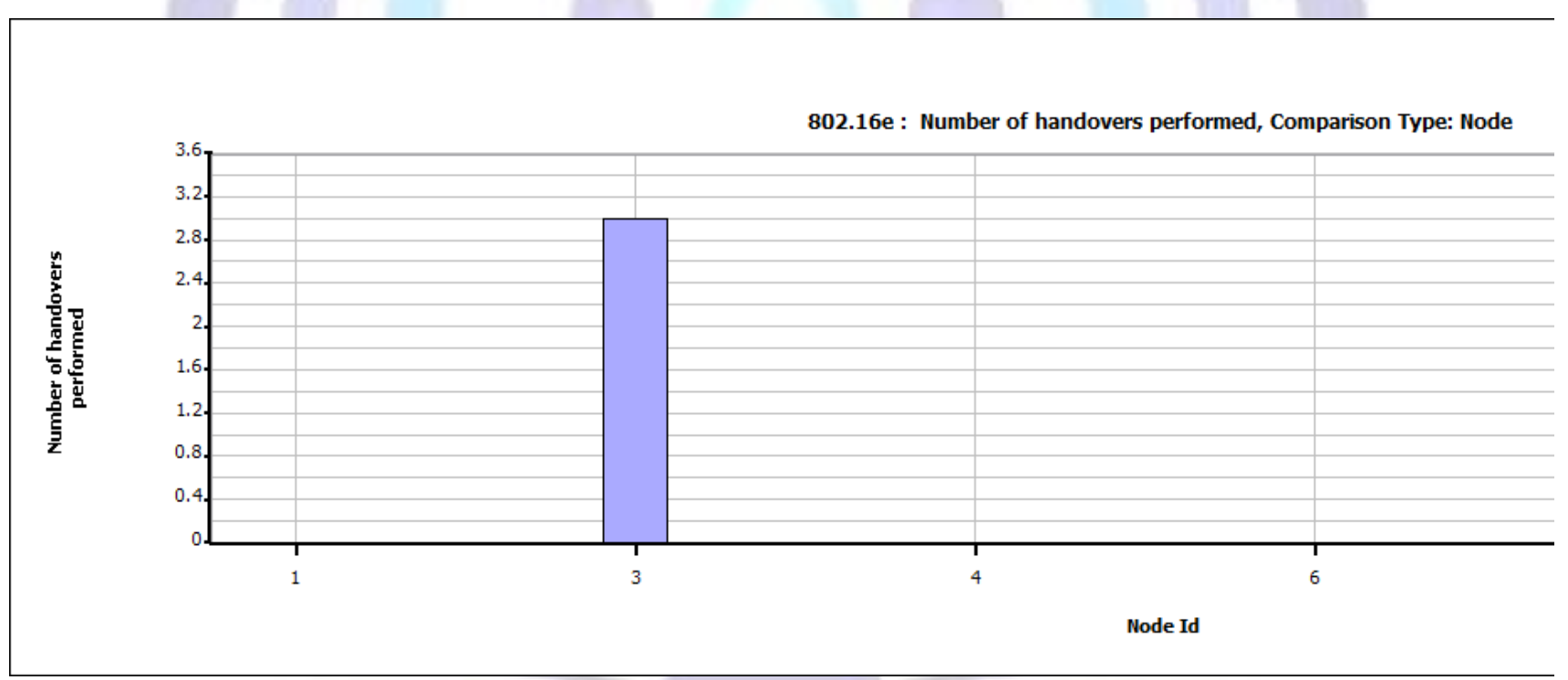

Figure 21: Number of Handover performed

There are 3 networks in the mobile WiMAX network which we considered for implementation .Node 3 performed 3 handover, one between network 1 and network 2, other one between 2 and 3, finally between 3 and 1 .

\section{CONCLUSION}

In this work the focus is on HARQ technique implementation using Qualnet simulator for efficient MAC to MAC Video transmission over mobile WiMAX.

After observing simulation results of number of data packet transmitted and received by $A R Q$ and $H A R Q$, we conclude that $H A R Q$ will receive more data packets when compare to $A R Q$ i.e $95 \%$ of transmitted data is received in $H A R Q$ compared to $82.97 \%$ in ARQ. Because $H A R Q$ uses combination of both FEC and ARQ. FEC will detect frequent error using error detecting algorithm whereas $A R Q$ will detect and correct less frequent error.

After observing simulation result of number of frames transmitted and received by ARQ and HARQ, we conclude that HARQ technique receives more frames than ARQ. This is due to the use of efficient scheduling algorithm i.e. $\mathrm{mmSIR}$ scheduler. $95 \%$ of transmitted frames are received in case of $\mathrm{HARQ}$ compared to $82.97 \%$ in $A R Q$. 
After observing simulation result of End to End delay, we conclude that delay will be more in $H A R Q$ technique, due to time taken for the retransmission of lost or error packets. HARQ retry time is used to fix the retransmission time, if the error packets are not received within this retry time, that packets are discarded. More delay is required to achieve good throughput.

After observing simulation result of Average jitter, we conclude that HARQ achieved less jitter compared to ARQ, this is due to less network congestion and less number of route changes.

After observing simulation result of Average throughput, we conclude that number of data transmitted per sec will be more in HARQ than ARQ. This is due to adaptive modulation and coding scheme at PHY layer, proper matching of QoS service classes and retransmission of lost packets. Nearly 3000 more packets are received per second by using HARQ.

With all these observation we come to conclusion that HARQ is more efficient for MAC to MAC video transmission over mobile WiMAX.

\section{FUTURE SCOPE}

1) Here we have used mmSIR scheduler, performance can also be improved further by other schedulers, this can be considered as a future work.

2) In this paper we authors limited HARQ implementation to fixed mobility path (flags, red colour in the WiMAX Scenario) in the mobile WiMAX scenario, the model can be extended for random mobility path.

3) Here we have considered non real time application, the method can be further extended for real time video application.

\section{ACKNOWLEDGMENTS}

The Authors would like to thank VGST (Vision Group on Science and Technology), Government of Karnataka, India for providing infrastructure facilities through the K-FIST level I project.

\section{REFERENCES}

[1]. Sunilkumar S Manvi, Mahabaleshwar S kakkasageri "Wireless and Mobile Networks concepts and protocols" Wiley India,2011

[2]. David Tse, P. Viswanath, "Fundamentals of Wireless communication", Cambridge, 2006.

[3]. Victoria Sgardoni, David Halls, Syed Mohsin Matloob Bokhari, David Bull and Andrew Nix, "Mobile WiMAX Video Quality and Transmission Efficiency", 2011 IEEE 22nd International Symposium on Personal, Indoor and Mobile Radio Communications.

[4]. Sheng Tzong ,Cheng,Chi Hsuan Wang,Gwo-Jiun Horng, "A cross layer approach of multimedia up streaming to ensure QoS in WiMAX", International Journal of Innovative Computing, Information and control volume 7,Number 11,November 2011.

[5]. Mehmet Sukru Kuran, Gurkan Gur, Tuna Tugcu, And Fatih Alagoz, "Applications of the Cross-Layer Paradigm for improving the performance of Wimax" Bogazici University.

[6]. R.V. Nee \& R. Prasad, "OFDM for WirelessMultimedia Communications” ArtechHouse Publishers, 2000.

[7]. $\quad$ Shu Lin \& Daniel J. Costello Jr."Error Control Coding”, Pearson /Prentice Hall, second Edition, 2004.

[8]. Seok Yee Tang,Peter Muller,Hamid R Sharif , "WiMAX security and Quality of Service”, Wiley India.2012

[9]. J. Roman, F. Berens, M. Kirsch, S. Tanrikulu "Hybrid ARQ schemes for future wireless systems based on MCCDMA," IST05.

[10]. Seyed Hossein, Hosseini Nazhad Ghazani, Jalil Jabari Lotf, "Bandwidth Efficient Cross-Layer Design Using Truncated Hybrid ARQ Approach for WiMAX Networks" ISSN 2090-4304 Journal of Basic and Applied Scientific Research.

[11]. J. Wang, M.Venkatachalam, Y. Fang, "System Architecture and Cross-Layer Optimization of video broadcast over WiMAX”, IEEE Journal on Sel. Areas in Communications, Vol. 25, No. 4, May 2007.

[12]. Aymen Belghith, Loutfi Nuaymi "Comparison of WiMAX scheduling algorithms and proposals for the rtPS QoS class".

[13]. O. Oyman, J. Foerster, Y. Tcha and S-C Lee, "Toward Enhanced Mobile Video Services over WiMAX and LTE", IEEE Communications Magazine, Aug. 2010.

[14]. Ali Alinejad, Nada Y. Philip \& Robert S. H. Istepanian, "Cross-Layer Ultrasound Video Streaming Over Mobile WiMAX and HSUPA Networks" IEEE TRANSACTIONS ON INFORMATION TECHNOLOGY IN BIOMEDICINE, VOL. 16, NO. 1, JANUARY 2012. 
[15]. H. Martikainen, A. Sayenko, O. Alanen and V. Tykhomyrov, "Optimal MAC PDU size in IEEE 802.16, IEEE ITNEWS 2008.

[16]. V. Sgardoni, M. Sarafianou, P. Ferre, A. Nix and D. Bull,"Robust video broadcasting over $802.11 \mathrm{a} / \mathrm{g}$ in time correlated fading channels", IEEE Trans. on Consumer Electronics, vol. 55, Issue 1, 2009.

[17]. WWW.WIMAXFORUM.ORG

[18]. WWW.SCALABLETECHNOLOGIES.COM

\section{Authors}

1. Arathi R Shankar: Associate Professor (PG studies)in the Department of Electronics andCommunicationat BMS College of Engineering, Bangalore India. She has received her BE (Telecommunication) and ME (Electronics) Degree from Bangalore University. She is currently pursuing her Ph.D from Visveswaraya Technological University, Karnataka, India. She has 20 years of teaching experience and her research interests include Wireless Communications and Antennas.

2. Raghavendra G: received the B.E degree in Electronics and Communication Engineering from Visveswaraya Technological University, Belgaum, India, in 2009. He is currently pursuing MTech. in Digital Communication at BMS College of Engineering, Bangalore, India. His research interest include Wireless Communication ,QoS control in WiMAX,Cross layer issue of WiMAX.

3. Dr. V. Sambasiva Rao: Professor in ECE Department of PES Institute of technology, Bangalore is an engineering graduate (1973) from College of Engineering, Kakinada, (Andhra University) and obtained Ph.D from BITS, Pilani in 2010. For over 37 years ( April 1974 to June 2011), he was associated with ISRO in various capacities and primarily responsible for the development of high bit rate data transmitters for all IRS series of satellites and various RF and microwave systems in S, $\mathrm{C}, \mathrm{X}, \mathrm{Ku}$ and $\mathrm{Ka}$ bands for IRS and INSAT missions.

He has successfully carried out numerous responsibilities as Project Manager/Deputy Project

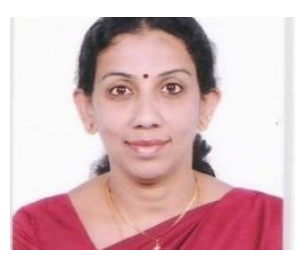
Director for different satellite projects and Deputy Director of Digital \& Communication Area in ISRO Satellite Centre. He is also responsible for various studies related to satellite technologies. Dr. Sambasiva Rao, a Fellow of IETE and a Member of Astronautical Society of India, has received six distinguished awards and published over 50 technical papers. 